\title{
Direkte Demokratie in der Schweiz - Länderbericht 2009/2010
}

Axel Tschentscher/Dominika Blonski

\section{Direkte Demokratie im Bund}

Die Instrumentenvielfalt der schweizerischen direkten Demokratie hat sich im Berichtszeitraum (Juni 2009 bis November 2010) formal verringert, weil die bereits voraussehbare Abschaffung der allgemeinen Volksinitiative nun auch offiziell vollzogen wurde (1.). International am heftigsten kommentiert wurde der Abstimmungserfolg der Minarettinitiative, zu der es inzwischen so viel aktuelle Literatur gibt, dass dieser Länderbericht auf die damit verbundenen Hintergründe konzentriert bleibt (2.). Ähnlich kontrovers, wenn auch international weniger beachtet, ging es bei der Todesstrafeninitiative und der Ausschaffungsinitiative zu (3.). Abgesehen davon sind wiederum zahlreiche Einzelabstimmungen durchgeführt (4.) und neue Initiativen und Referenden lanciert worden (5.).

\section{Abschaffung der, ,allgemeinen Volksinitiative“}

Mit dem Bundesbeschluss über den Verzicht auf die Einführung der allgemeinen Volksinitiative wurde die beabsichtigte Erweiterung der Volksrechte auf Bundesebene wieder zurückgenommen. Die vorbehaltlich der Ausführungsgesetzgebung bereits vollzogene Änderung der Verfassung wurde rückgängig gemacht. Beim obligatorischen Referendum über diese Verfassungsänderung stimmten mehr als zwei Drittel der Teilnehmenden und sämtliche Kantone für die Abschaffung des missglückten Instruments (27.9.2009; .../cr/2008/20080641.html). Dabei war die Intention für dessen Einführung zunächst allgemein akzeptiert worden. Es sollte den direktdemokratischen Mitteln auf Bundesebene ein Initiativrecht hinzugefügt werden, mit dem politische Anliegen direkt auf Gesetzesstufe verwirklicht werden können. In den Kantonen besteht diese Möglichkeit bereits. Im Bund hätte sie den zusätzlichen Vorteil gehabt, dass die durch solche Gesetzesinitiativen bewirkten Rechtsänderungen einer verfassungskonformen und völkerrechtskonformen Auslegung zugänglich wären. 
Gescheitert ist die allgemeine Volksinitiative letztlich an Verfahrensfragen. Der bundesrätliche Entwurf für ein Ausführungsgesetz war sowohl vom Nationalrat (2006) als auch vom Ständerat (2007) schroff zurückgewiesen worden (Nichteintretensentscheid). Schon die vorausgegangene Vernehmlassung und die öffentliche politische Diskussion über den Entwurf hatten zu dem allgemeinen Eindruck geführt, hier werde ein kompliziertes und zeitraubendes Vorgehen nötig, das die Gesamtheit der politischen Instrumente für das Volk eher unattraktiver als attraktiver mache. Anders als in den Kantonen lässt sich im Bund nämlich nicht ohne weiteres eine Vereinfachung der Abläufe erreichen, denn im Zweikammerparlament muss jede Kammer in die Diskussion über die Umsetzung und über einen möglichen Gegenentwurf einbezogen werden. Außerdem sollte bereits als Teil des Verfahrens die Beschwerde gegen die inhaltliche Umsetzung der allgemeinen Volksinitiative möglich sein, was zusätzliche Verfahrensschritte vor dem Bundesgericht bedingt hätte. ${ }^{1}$ Insgesamt wäre das Instrument dadurch wenig bürgerfreundlich ausgefallen. Mit dem geradezu peinlichen Scheitern der Politik bei diesem Reformvorhaben dürfte die Diskussion über eine Erweiterung der direktdemokratischen Instrumente auf Bundesebene für längere Zeit beendet sein.

Politikwissenschaftlich gilt diese Abstimmung als Paradebeispiel für einen Elitenkonsens. ${ }^{2}$ Regierung, Parlament, Parteien und Verbände waren fast ausnahmslos einer Meinung. Eine öffentliche Diskussion oder gar ein Abstimmungskampf fand darum nicht statt. Mit einer Zustimmungsrate von 67,9\% wurde die Abschaffung des Instruments fast gleich stark befürwortet wie wenige Jahre zuvor seine Einführung (2003: 70,4\%). Unter solchen Umständen treten Effekte auf, die sonst für Abstimmungen ungewöhnlich sind. So gaben $23 \%$ der nachträglich Befragten an, dass sie ohne eigene Meinungsbildung einfach der Empfehlung von Parteien, Verwandten oder Regierung gefolgt seien. Fast ein Drittel der Zustimmenden berücksichtigte solche Empfehlungen in irgendeiner Weise. Gemessen am zurückliegenden Zehnjahreszeitraum ist das ein Rekordwert. ${ }^{3}$ Unter denjenigen, die gegen die Abschaffung der allgemeinen Volksinitiative stimmten, fand sich zudem ein außerordentlich großer Anteil von Personen,

$1 \mathrm{Zu}$ den komplizierten Details des vorgeschlagenen Verfahrens siehe: Botschaft über die Einführung der allgemeinen Volksinitiative und über weitere Änderungen der Bundesgesetzgebung über die politischen Rechte vom 31.5.2006, BBl 2006, 5261 (.../ff/2006/5261. pdf).

2 T. Milic/S. Kuster/T. Widmer, Bundesbeschluss vom 19.12.2008 über den Verzicht auf die Einführung der allgemeinen Volksinitiative, VOX-Analyse, hrsgg. von gfs.bern, 2009, S. 21 (21).

3 Milic/Kuster/Widmer, VOX-Analyse (Fn. 2), S. 26. 
die aus falschen Motiven handelten. Sie rechtfertigten ihre Ablehnung mit Gründen, die eigentlich zur Zustimmung der Abschaffung hätten führen müssen, etwa mit dem Argument, das Instrument sei nicht umsetzbar oder es sei zu kostspielig. Diese Analysen zeigen, wie wichtig ein inhaltsreicher öffentlicher Diskurs für das Funktionieren der direkten Demokratie ist. Fällt die vorherige öffentliche Auseinandersetzung mit dem Thema aus, so kann das Volk weder einen Kontrapunkt zur institutionalisierten Politik setzen, noch können die einzelnen Stimmberechtigten eine informierte und dadurch autonome Entscheidung treffen.

\section{Völkerrechtswidrige Volksinitiativen am Beispiel der Minarettverbotsinitiative}

Keine andere Initiative hat im Berichtszeitraum so hohe Wellen geschlagen wie die Minarettverbotsinitiative. ${ }^{4}$ Besonderes Aufsehen im In- und Ausland erregte ein Plakat, auf dem vor raketenförmigen, gänzlich schwarzen Minaretten eine tief verschleierte muslimische Frau als schwarze Figur abgebildet war - beides finster dominant über einer darunter liegenden Schweizerfahne. Als am 29.11.2009 nach emotional geführtem Abstimmungskampf eine überraschend starke Mehrheit von Volk und Ständen für die Annahme des Minarettverbots stimmte (57,5\% und 17 5/2 Kantone), war die institutionalisierte Politik, die mit Ausnahme der SVP und EVU dagegen votierte, geradezu überrollt. Kurz zuvor hatte eine vom schweizerischen Rundfunk (SRG) in Auftrag gegebene Umfrage eine Ablehnung durch 53\% prognostiziert. Mit großem Aufwand wurde nun der internationalen Öffentlichkeit die Relativität des Ergebnisses erläutert: nicht für Moscheen gelte das Verbot, sondern nur für Minarette; zahlreiche Moscheen (ca. 160) bestünden bereits für die große Zahl von Muslimen in der Schweiz (ca. 400.000); vereinzelt gebe es in der Schweiz Minarette (4), deren Bestand nicht betroffen sei; die Abstimmung sei ein Nebeneffekt der generell kritischen Stimmung gegenüber nicht integrierten Ausländern (ca. 80\% der in der Schweiz lebenden Muslime sind Ausländer) und kein Votum gegen die Religionsfreiheit. Tatsächlich zeigt auch die nachträgliche Befragung der Abstimmenden, dass die Einstellung gegenüber Ausländern mit dem Abstimmungsverhalten korreliert.

4 Neuer Art. 72 Abs. 3 BV („Der Bau von Minaretten ist verboten.“); eingereicht am 8.7.2008; Botschaft vom 27.8.2008 ohne Gegenvorschlag; .../vi/vis353.html. Zu den vorgängigen Verfahrensphasen und zur Diskussion siehe A. Tschentscher, Direkte Demokratie in der Schweiz - Länderbericht 2008/2009, in: L.P. Feld u.a. (Hrsg.), Jahrbuch für direkte Demokratie 2009, 2010, S. 205 (219 ff., 220). 
Wer allgemein die Auffassung vertritt, die ausländische Bevölkerung solle weniger Rechte haben als Schweizerinnen und Schweizer, der stimmte zu 84\% für das Minarettverbot. ${ }^{5}$

Offenbar handelte es sich um eine symbolische Abstimmung. Das am häufigsten genannte Motiv für ein Ja zum Minarettverbot bestand darin, ein Zeichen gegen die empfundene Islamisierung der Schweiz und Europas setzen zu wollen. Im Rückblick wird die Abstimmung darum von der Politikwissenschaft in die Reihe der eher seltenen Symbol-Initiativen gestellt, bei denen der unmittelbare Entscheidungsgegenstand weniger Gewicht hat als die mittelbare Kritik an einer allgemeineren Entwicklung - etwa die Armeebrieftaubeninitiative (1996) als Zeichen gegen die Technisierung der Armee und das Mobilfunkantennen-Moratorium (2003) als Zeichen gegen den grassierenden Handyismus. ${ }^{6}$ Während solche Initiativen allerdings meist im Unterschriftenstadium scheitern, hatte die Minarettverbotsinitiative durchschlagenden Erfolg. Will man eine vergleichbar erfolgreiche symbolische Initiative finden, muss man mehr als ein Jahrhundert zurückreichen. Die Eidgenössische Volksinitiative „für ein Verbot des Schlachtens ohne vorherige Betäubung“", die zwar von Tierschutzvereinen eingereicht worden war, sich aber vor allem symbolisch gegen Juden richtete, wurde am 20.8.1893 mit vergleichbar deutlicher Mehrheit angenommen (60,1\% und 10 3/2 Stände).

Ein Bericht des Bundesrates zum Verhältnis von Völkerrecht und Landesrecht, der schon seit längerer Zeit aufgrund parlamentarischer Aufträge in Arbeit war, erhielt durch die Minarettinitiative zusätzliche Aufmerksamkeit. ${ }^{8}$ Die Landesregierung konstatiert darin, dass seit den 1990er Jahren vermehrt Volksinitiativen eingereicht worden seien, die gegen das Völkerrecht verstoßen, aber gleichwohl für gültig zu erklären sind, weil sie nicht dessen zwingende Normen (insbesondere Völkermord, Sklaverei, Folter) verletzen. Der Frage, wie man in solchen Fällen eine völkerrechtskonforme Umsetzung erreichen könne, ohne den

5 H. Hirter/A. Vatter, Analyse der eidgenössischen Abstimmungen vom 29. November 2009, VOX-Analyse, hrsgg. von gfs.bern, 2010, S. 25 (26), mit weiteren Analyseergebnissen.

6 O. Sigg, Eine Symbol-Initiative über die gar nicht diskutiert wurde. Die Vorlage zum Minarettverbot ist in der Landesgeschichte eine Ausnahme, in: NZZaS v. 24.1.2010, S. 14.

7 Einführung eines neuen Artikels $25^{\text {bis }}$ in die Bundesverfassung: „Das Schlachten der Tiere ohne vorherige Betäubung vor dem Blutentzuge ist bei jeder Schlachtart und Viehgattung ausnahmslos untersagt.“

8 Bundesrat (Hrsg.), Das Verhältnis von Völkerrecht und Landesrecht. Bericht vom 5.3.2010 (.../ff/2010/2263.pdf). 
Willen der Initianten zu verletzen, komme durch die Minarettverbotsinitiative besondere Aktualität zu. Es sei sogar die Neuaushandlung oder Kündigung von entgegenstehenden völkerrechtlichen Verträgen zu erwägen. Im Ergebnis erteilt der Bundesrat den bisherigen Reformvorschlägen (konsultative Kontrolle, obligatorischer Gegenentwurf, Undurchführbarkeitsvorbehalt, erweiterte Ungültigkeitsgründe) vorläufig eine Absage. Die besonders vordringliche Frage einer Ausweitung materieller Schranken der Verfassungsrevision reicht er als Abklärungsauftrag an die zuständigen Bundesämter weiter.

\section{Weitere Kontroversen}

a) Todesstrafeninitiative (faktischer Rückzug)

Großes Aufsehen erregte die Volksinitiative „Todesstrafe bei Mord mit sexuellem Missbrauch“.9 In einer verfassungstechnisch sehr konkreten und überlegten Weise zeigten die Initianten einen Weg für die Wiedereinführung der Todesstrafe auf. Zwischen Lebensrechtsgarantie und Todesstrafenverbot (Art. 10 Abs. 1 Satz 1 und 2 BV) wurde dazu ein neuer Ausnahmetatbestand eingefügt: „Jeder Mensch hat das Recht auf Leben. Wer in Kombination mit einer sexuellen Handlung mit einem Kind, sexueller Nötigung oder Vergewaltigung eine vorsätzliche Tötung oder einen Mord begeht, verliert sein Recht auf Leben und wird mit dem Tod bestraft. In allen anderen Fällen ist die Todesstrafe verboten." Sogar an eine Übergangsbestimmung und an eine Verfahrensvorschrift war gedacht worden. Letztere war so geschickt formuliert, dass im Annahmefalle eine direkte Anwendbarkeit der Regelung ohne Dazwischentreten des Gesetzgebers, ohne Relativierungen und ohne Verzögerungen garantiert gewesen wäre (Art. 123a Abs. 4 BV): ,Wer in Kombination mit einer sexuellen Handlung mit einem Kind, sexueller Nötigung oder Vergewaltigung eine vorsätzliche Tötung oder einen Mord begeht, wird hingerichtet, unabhängig von Gutachten oder wissenschaftlichen Erkenntnissen. Der Bund vollzieht die Hinrichtung. Die Hinrichtung wird innerhalb von drei Monaten, nachdem die Verurteilung rechtskräftig geworden ist, vollzogen. Das Gericht legt das Hinrichtungsdatum und die Hinrichtungsmethode fest.“

9 Geänderter Art. 10 Abs. 1 und 3 BV, neuer Art. 123a Abs. 4 BV sowie neue Übergangsbestimmung $\mathrm{zu}$ Art. 10 Abs. 1 und 3 und Art. 123a Abs. 4 BV; Sammelfrist bis 24.2.2012; .../vi/vis392.html. 
Nach den Erfahrungen mit der Unverjährbarkeitsinitiative, die in ähnlicher Weise aus persönlicher Betroffenheit an die tief empfundene Abscheu vor Sexualverbrechen appelliert hatte und damit bei der Abstimmung erfolgreich war, wurde die Todesstrafeninitiative sehr ernsthaft diskutiert. Dabei spielte eine weitere Besonderheit der eidgenössischen Volksinitiative eine Rolle: die offizielle Publikation der Vorprüfung. Bei diesem ersten Verfahrensschritt nach Einreichung einer Initiative prüft die Bundeskanzlei, ob die gesetzlichen Formvorschriften eingehalten sind. Die Unterschriftenlisten müssen Titel, Wortlaut, Publikationsdaten, Rückzugsklausel und Strafbarkeitswarnung sowie die richtige Anzahl von Urheberinnen (mindestens sieben, höchstens 27) mit Namen und Adressen enthalten. In diesem Fall bestand das Initiativkomitee aus den mindestens geforderten sieben Personen, die erkennbar aus drei Familien stammten und letztlich durch eine Einzelperson, einen 35-jährigen, nicht politisch prominenten Bankangestellten, nach außen vertreten wurden. Er hatte die Initiative lanciert, weil sich das Strafverfahren im Mordfall an seiner Schwägerin seit eineinhalb Jahren hinzog.

In diesem Stadium des Verfahrens findet keine inhaltliche Vorprüfung statt. Gleichwohl wird das Ergebnis als Verfügung der Bundeskanzlei über eine erfolgreiche Prüfung öffentlich im Bundesblatt publiziert. Dadurch gewann die Initiative in den Medien eine Bedeutung, die ihr nach dem organisatorischen Hintergrund gar nicht gebührte. Die wenigen Privatpersonen konnten, unterstützt durch eine Internetpräsenz (www.todes-strafe.ch), nach außen den Eindruck einer gewichtigen politischen Initiativbewegung erzeugen. Sofort nach Bekanntwerden der Verfügung waren Zeitungen und Rundfunkprogramme gefüllt mit Rückblicken auf die letzten strafrechtlichen und militärstrafrechtlichen Vollstreckungen der Todesstrafe in der Schweiz (1940 bzw. 1944) und mit vergleichenden Analysen zur Todesstrafenpraxis in der Welt.

Die rechtsdogmatische Diskussion entbrannte einerseits über bestehende und neue Ungültigkeitsgründe der Volksinitiative, ist doch die Wiedereinführung der Todesstrafe ein so klarer Verstoß gegen die von der Schweiz unterzeichneten Zusatzprotokolle 6 und 13 der Europäischen Menschenrechtskonvention, dass sie als Aufkündigung des europäischen Konsenses angesehen werden muss. Die Schweiz hätte mindestens diese Protokolle kündigen müssen und sich innereuropäisch mit Weißrussland in eine Außenseiterposition begeben, was politisch als unvorstellbar gilt. Gleichzeitig kann die Todesstrafe nicht dem zwingenden Völkerrecht zugeordnet werden, weil insoweit gerade kein weltweiter Konsens besteht. Auch grundrechtsdogmatisch hatte der Vorstoß erhebliche Sprengkraft, denn das Verbot der Todesstrafe ist in der Schweiz als Kerngehalt des Lebensrechts anerkannt. Bei Annahme der Initiative wäre darum ein Präzedenzfall ent- 
standen, der beweist, dass nicht einmal die grundrechtlichen Kerngehalte wirksam vor den Volksrechten geschützt sind.

Mit dem sofortigen Rückzug der Scheininitiative nur einen Tag nach der Veröffentlichung im Bundesblatt präsentierten die Initianten ein taktisches Novum, das es bisher so noch nie gegeben hatte. Im Ergebnis lösten sie das Medienecho aus, ohne mit der aufwendigen Unterschriftensammlung auch nur zu beginnen. Sie begründeten ihren Rückzug damit, dass sie erstens in der aus ihrer Sicht täterzentrierten Strafrechtsdebatte der Schweiz die Opferperspektive wieder zur Geltung bringen wollten, was schon in wenigen Tagen geglückt sei, und zweitens selbst überrascht und erschrocken über die heftigen Reaktionen und teilweise sogar Drohungen waren, denen sie persönlich ausgesetzt waren. Die Bundeskanzlei nahm öffentlich zu diesem taktischen Schachzug Stellung. Für eine Buße wegen Missbrauchs der Volksrechte fehle eine Grundlage. Auch eine Gebühr sei mit der Ausübung der Rechte nicht verbunden. Und de lege ferenda empfehle es sich auch nicht, nur wegen eines Einzelfalls die Verfahrensregeln über die Ausübung der Volksrechte zu ändern.

Genau betrachtet gibt es zwei Formen des Rückzugs, den offiziellen und den lediglich medial-faktischen. Den offiziellen Rückzug erklärt ein Initiativkomitee mit absoluter Mehrheit seiner Mitglieder verbindlich gegenüber der Bundeskanzlei (Art. 73 Abs. 1 BPR), woraufhin das Geschäft schon vor Ablauf der Sammelfrist abgeschrieben wird. Darüber führt die Bundesverwaltung eine öffentliche Liste. ${ }^{10}$ Ein solcher Rückzug ist bei der Todesstrafeninitiative allerdings nicht erfolgt. Statt dessen wurde gegenüber den Medien erklärt, man ziehe die Initiative zurück. Rechtlich führt das erst dann zum Ende des Verfahrens, wenn die Sammelfrist verstreicht, ohne dass 100.000 gültige Unterschriften eingereicht wurden. Beginnend mit der Veröffentlichung im Bundesblatt (24.8.2010) wäre also theoretisch noch bis zum 24.2.2012 (18 Monate gemäß Art. 71 Abs. 1 BPR) die Unterschriftensammlung und -einreichung möglich. Tatsächlich ist durch den Verlauf der öffentlichen Diskussion der Impetus der Initiative bereits verbraucht, so dass ein Wiederaufgreifen faktisch unmöglich sein dürfte. Selbst bei den Befürwortern von Strafrechtsverschärfungen hat die Initiative zudem keine nennenswerte Unterstützung gewinnen können. Insoweit hebt sie sich von den anderen völkerrechtswidrigen Volksinitiativen der jüngeren Zeit (Verwahrungsinitiative, Unverjährbarkeitsinitiative, Minarettverbotsinitiative, Ausschaffungsinitiative) durch ihre unrealistische Zuspitzung ab. Ihre Bedeutung liegt rückblickend darin, die äußersten Grenzen der verfassungstechnischen Veränderung durch 
Volksinitiativen ausgetestet und das Verfahren dieses Instruments erstmalig bis zur Grenze des Missbrauchs strapaziert zu haben.

b) Ausschaffungsinitiative (28. November 2010)

Die Volksinitiative „Für die Ausschaffung krimineller Ausländer (Ausschaffungsinitiative) ${ }^{\text {، } 11}$ wurde nach einem taktisch schwer durchschaubaren Abstimmungskampf bei deutlich überdurchschnittlicher Stimmbeteiligung (53\%) von den Initianten klar für sich entschieden (52,9\% und 15 5/2 Stände). Dabei stand ursprünglich selbst die Gültigerklärung in Frage, denn der Initiativtext kannte keine Ausnahme für Folterstaaten. Weil das Non-Refoulement-Prinzip anerkannter Bestandteil des zwingenden Völkerrechts ist, wäre dieses kaum je einschlägige Kriterium der materiellen Schranken hier ausnahmsweise einmal relevant gewesen. In der Debatte über die Gültigerklärung konnten die Befürworter der Initiative allerdings erfolgreich geltend machen, dass nach dem Wortlaut der Verfassungsänderung nur das „Aufenthaltsrecht sowie alle Rechtsansprüche auf Aufenthalt in der Schweiz" entfallen würden. Die Grenze des RefoulementVerbots sei keine Frage des Aufenthaltsrechts, sondern des Vollzugs einer Ausweisung, die sich in einem separaten Schritt anschließe und insofern durch die Verfassungsänderung gar nicht betroffen sein könne.

Indem die Initiativgegner im Parlament einen Gegenentwurf zur Abstimmung stellten, begingen sie rückblickend einen taktischen Fehler. Dieser Entwurf wurde vom Volk deutlich abgelehnt (54,2\%). Die genaue Analyse der Gründe steht noch aus, doch maßgeblich dürfte die Spaltung der Gegnerschaft gewesen sein. Der Gegenentwurf nahm seinerseits so viel vom Verschärfungsanliegen der Initianten auf, dass er eine politische Bewegung für das doppelte Nein auslöste. In Ermangelung einer klaren Alternative konnte so der Initiativvorschlag zusätzlich Profil gewinnen. Er wurde im Ergebnis nur noch in den großen Städten und in der Romandie abgelehnt, sonst hingegen überall angenommen (sog. Röstigraben und Stadt-Land-Graben).

Da die Initiative nicht direkt anwendbar ist, steht jetzt die Umsetzung im Parlament bevor, die parallel zu den Wahlen im kommenden Jahr stattfindet. Dies allein dürfte bereits polarisierend wirken. Vor allem aber wird sich die Problematik der Verwahrungsinitiative wiederholen, bei der letztlich keine völker-

11 Neuer Art. 121 Abs. 3-6 BV sowie neue Übergangsbestimmung zu Art. 121 BV; eingereicht am 15.2.2008; Botschaft vom 24.6.2009 mit indirektem Gegenvorschlag; .../vi/vis357.html. 
rechts- und verfassungskonforme Umsetzung gefunden wurde, die das Anliegen der Initianten tatsächlich vollständig aufgreift. Indem der Initiativtext von einem relativ wahllosen Deliktskatalog ausgeht, nach dem beispielsweise auch jeder Einbruchdiebstahl und Sozialmissbrauch ohne Schwereprüfung im Einzelfall das Aufenthaltsrecht zum Erlöschen bringt, dürfte eine vollständige Umsetzung nicht möglich sein, ohne gleichzeitig gegen das Verhältnismäßigkeitsprinzip zu verstoßen.

\section{Abstimmungspraxis}

a) Forschung am Menschen (7. März 2010)

Der von der Bundesversammlung ausgearbeitete Verfassungsartikel über die Forschung am Menschen (neuer Art. 118b BV; beschlossen am 25.9.2009; .../cr/2007/20071024.html) erteilte dem Bund eine umfassende Zuständigkeit zur Regelung der Forschung am Menschen. Die wegen des obligatorischen Referendums am 7.3.2010 stattgefundene Volksabstimmung zeigte mit nur 22,8\% NeinStimmen eine deutliche Zustimmung des Volkes zum Vorhaben. Dieses Ergebnis war allerdings nicht sonderlich überraschend, hatte man doch alle kontroversen Kriterien nach jahrelanger Diskussion aus dem Verfassungstext verbannt und in Formelkompromisse überführt. So vereint die Zweckbestimmung des Verfassungszusatzes jetzt alle potentiell konfligierenden Anliegen: den Schutz der Würde und Persönlichkeit des Menschen in der Forschung ebenso wie die Forschungsfreiheit und die Bedeutung der Forschung für Gesundheit und Gesellschaft. An die Stelle strikter Grenzziehungen treten nur noch generalklauselartig formulierte Grundsätze, etwa dass die Risiken nicht in einem Missverhältnis zum Nutzen stehen sollten (Art. 118b Abs. 2 Bst. b BV). Bei den umstrittenen Grenzen, die im Forschungsartikel nur noch angedeutet sind (Einwilligungserfordernis, Forschung mit urteilsunfähigen Personen), finden sich zudem Ausnahmeklauseln und Abwägungsformeln (z.B. Art. 118b Abs. 2 Bst. c BV: „Belastungen nur minimal"). Die eigentlichen Kontroversen werden dadurch vom obligatorischen Referendum ferngehalten und dem Gesetzgebungsverfahren zugeleitet, bei dem mit dem fakultativen Referendum eine weniger intensive Direktbeteiligung des Volkes besteht. 
b) Steuergerechtigkeits-Initiative (28. November 2010)

Ziel der Volksinitiative „Für faire Steuern. Stopp dem Missbrauch beim Steuerwettbewerb (Steuergerechtigkeits-Initiative)“ war die Einschränkung der kantonalen Steuerautonomie sowie des kantonalen Steuerwettbewerbs (neuer Art. 129 Abs. $2^{\text {bis }}$ BV sowie neue Übergangsbestimmungen zu Art. 129 Abs. $2^{\text {bis }}$ und neue Übergangsbestimmung zu Art. 135 BV; eingereicht am 6.5.2008; .../vi/vis349.html). Bei der Volksabstimmung am 28.11.2010 folgte das Volk der Empfehlung des Bundesrates und der Bundesversammlung und sprach sich mit einem Nein-Stimmenanteil von 58,5\% deutlich gegen die Initiative aus. Die Abstimmung ist ein weiteres Beispiel dafür, dass der Steuerwettbewerb unter den Kantonen im Volk eine breite und sehr stabile Unterstützung erfährt. Obgleich einzelne Kantone sich in der jüngeren Vergangenheit mit einem regelrechten Steuersatzdumping gegenseitig unterboten haben, wird an dem Prinzip des Wettbewerbs, das sogar bis auf die Gemeindeebene hinunterreicht, bisher nicht gerüttelt.

c) Weitere Volksabstimmungen

In chronologischer Reihenfolge fanden im Übrigen folgende Abstimmungen auf Bundesebene statt:

Das Vorhaben der Bundesversammlung, für einen siebenjährigen Zeitraum (2011 bis 2017) die Invalidenversicherung durch Anhebung der Mehrwertsteuersätze zusätzlich zu finanzieren, unterstand dem obligatorischen Referendum, weil unter anderem eine Verfassungsänderung vorgesehen war (neue Übergangsbestimmung zu Art. 130 Mehrwertsteuer; beschlossen am 13.6.2008; .../cr/2005/20051235.html). Das Ausführungsgesetz unterstand demgegenüber nur dem fakultativen Referendum, das nicht ergriffen wurde. Trotzdem wäre das Gesetz, weil sein Inkrafttreten mit der Verfassungsänderung inhaltlich verbunden wurde, ohne Zustimmung von Volk und Ständen nicht wirksam geworden (.../geschaefte.aspx?gesch_id=200500-53). Diese Zustimmung zu dem Vorhaben erteilte das Volk am 27.9.2009 mit relativ deutlicher Mehrheit von 54,6\% JaStimmen.

Die aufgrund des obligatorischen Referendums am 29.11.2009 stattgefundene Volksabstimmung zur Schaffung einer Spezialfinanzierung für Aufgaben im Luftverkehr fiel mit einem deutlichen Ja-Stimmenanteil von $65 \%$ zu Gunsten der Absicht der Bundesversammlung aus. Ziel der Verfassungsänderung war die künftige Verwendung von Mineralölsteuererträgen auf Flugtreibstoffen im Sinne 
der Luftfahrt (geänderter Art. 86 Abs. 3, $3^{\text {bis }}$ und 4 BV; beschlossen am 3.10.2008; .../cr/2006/20061794.html).

Die Volksinitiative „für ein Verbot von Kriegsmaterial-Exporten“ wollte die Ausfuhr von Waffen verbieten (neue Art. 107 Abs. 3 und 107a BV sowie neue Übergangsbestimmung zu Art. 107a BV; eingereicht am 21.9.2007; .../vi/vis346.html). Bundesrat und Bundesversammlung empfahlen die Initiative zur Ablehnung. Vom Volk wurde diese Ablehnung am 29.11.2009 mit einem deutlichen Nein-Stimmenanteil von $68,2 \%$ ausgesprochen.

Mit der Volksinitiative „Gegen Tierquälerei und für einen besseren Rechtsschutz der Tiere (Tierschutzanwalt-Initiative)“ wurde unter anderem die Institution eines Tierschutzanwaltes vorgesehen (neuer Art. 80 Abs. 4 und 5 BV; eingereicht am 26.7.2007; .../vi/vis340.html). Das Volk folgte der Empfehlung des Bundesrates und der Bundesversammlung und lehnte das Ansinnen am 7.3.2010 mit einem Nein-Stimmenanteil von 70,5\% klar ab.

Die Bundesversammlung beschloss die Änderung des Bundesgesetzes über die berufliche Alters-, Hinterlassenen- und Invalidenvorsorge (BVG) mit dem hauptsächlichen Ziel, den Mindestumwandlungssatz in vier Teilschritten ab 1.1.2008 bis zum Erreichen von 6,4\% per 1.1.2011 zu senken (geänderte Art. 13 Abs. 1, Art. 14 Abs. 2 und 3, Art. 16 und Art. 24 Abs. 2 BVG; beschlossen am 19.12.2008; .../cr/2006/20062073.html). Der Mindestumwandlungssatz ist der tiefste Prozentsatz des angesparten Kapitals, der den Pensionierten als Rente jährlich ausbezahlt wird. Die Gesetzesänderung unterstand dem fakultativen Referendum, welches am 6.5.2009 zustandegekommen war und am 7.3.2010 zu einer Volksabstimmung führte, in der sich das Volk mit einem hohen NeinStimmenanteil von 72,7\% schließlich gegen die Änderung aussprach.

Mit der Änderung des Bundesgesetzes über die obligatorische Arbeitslosenversicherung und die Insolvenzentschädigung (Arbeitslosenversicherungsgesetz, AVIG) wollte die Bundesversammlung den normalen Beitragssatz von 2 auf 2,2\% erhöhen sowie die Leistungen um rund 500 Mio. Franken reduzieren. Zur Entschuldung sollte zudem der Beitragssatz zeitlich befristet von 2,2 auf 2,3\% erhöht und ein Solidaritätsbeitrag von 1 Prozent eingeführt werden (Änderung zahlreicher Bestimmungen des AVIG; beschlossen am 19.3.2010; .../cr/2008/ 20081668.html). Gegen diese Änderung wurde das fakultative Referendum ergriffen und kam am 12.7.2010 zustande. Das Stimmvolk sprach sich bei der Volksabstimmung am 26.9.2010 mit knappen 53,4\% für die Änderung aus. 
5. Hängige Volksinitiativen und Referenden

a) Initiierungsphase

Während der Unterschriftensammlung lassen sich die im Rahmen der Vorprüfung genehmigten Unterschriftenlisten auf den Aktualitätsseiten der Bundeskanzlei abrufen (http://www.admin.ch/ch/d/pore/vi/). In diesem frühen Stadium befinden sich derzeit (Anfang Dezember 2010)

- die Volksinitiative „Für ein EU-Beitrittsmoratorium“, die während zehn Jahren EU-Beitrittsverhandlungen für die Schweiz nicht zulassen will (allgemeine Anregung; Sammelfrist bis 23.5.2012; .../vi/vis395.html),

- die Volksinitiative „Bürokratie-Stopp“, welche einerseits die Verankerung des Rechts auf wenig Bürokratie als Grundrecht in der Bundesverfassung fordert, andererseits die Exekutive und Legislative verpflichtet, insbesondere auf KMU Rücksicht zu nehmen und Regelungsdichte sowie administrative Belastung möglichst gering zu halten (neue Art. 9a und Art. 94 Abs. 3 zweiter Satz BV; Sammelfrist bis 12.4.2012; .../vi/vis394.html),

- die Volksinitiative „Für Transparenz in der Krankenversicherung (Schluss mit der Vermischung von Grund- und Zusatzversicherung)“, welche es Krankenversicherern verbieten möchte, neben der obligatorischen Krankenversicherung nach KVG auch Zusatzversicherungen nach VVG anzubieten (neuer Art. 117 Abs. 3 BV; Sammelfrist bis 28.3.2012; .../vi/vis-393.html),

- die „Stipendieninitiative“, die das bisher kantonal geregelte Stipendienwesen für Ausbildungsbeiträge an Studierende von Hochschulen auf Bundesebene harmonisieren will (geänderter Art. 66 BV sowie neue Übergangsbestimmung zu Art. 66 BV; Sammelfrist bis 20.1.2012; .../vi/vis390.html),

- die Volksinitiative „Ja zur Aufhebung der Wehrpflicht“, welche die Aufhebung der Wehrpflicht und die Freiwilligerklärung des Zivildienstes als Ziel verfolgt (geänderter Art. $59 \mathrm{BV}$ sowie neue Übergangsbestimmungen zu Art. 59 BV; Sammelfrist bis 6.1.2012; .../vi/vis391.html),

- die Volksinitiative „Wenden wir die Menschenrechte an auf Frauen und Männer = Schweiz", die die Allgemeine Erklärung der Menschenrechte der UNO zu einem Teil der Schweizerischen Verfassung erklären will (neuer Art. 8a BV; Sammelfrist bis 19.11.2011; .../vi/vis389.html),

- die Volksinitiative „Für ein bedingungsloses Grundeinkommen finanziert durch Energielenkungsabgaben“, welche die Finanzierung von Grundeinkommen und Sozialversicherungen durch Energielenkungsabgaben vorsieht (neuer Art. 41 Abs. 1 Bst. h-j BV; Sammelfrist bis 19.11.2011; .../vi/vis388.html),

- die Volksinitiative „Schutz vor Rasern“, die höhere Strafen für die Verletzung von Verkehrsregeln - insbesondere bezüglich Geschwindigkeitsvor- 
schriften - in der Verfassung verankern will (neuer Art. 123c BV; Sammelfrist bis $27.10 .2011 ; . . /$ vi/vis384.html),

- die Volksinitiative „Schluss mit der MwSt-Diskriminierung des Gastgewerbes!“, welche eine Anpassung des MwSt-Satzes von im Gastgewerbe konsumierten Speisen an jenen von in Läden gekauften Lebensmitteln fordert (neuer Art. 130 Abs. $1^{\text {bis }}$ BV sowie neue Übergangsbestimmung zu Art. 130 Abs. $1^{\text {bis }}$ BV; Sammelfrist bis 7.10.2011; .../vi/vis386.html),

- die Volksinitiative „Neue Arbeitsplätze dank erneuerbaren Energien (Cleantech-Initiative)“, die die Schaffung von Arbeitsplätzen durch die Förderung von erneuerbaren Energien verlangt (neuer Art. 89 Abs. $1^{\text {bis }}$ und $2^{\text {bis }} \mathrm{BV}$, geänderter Art. 89 Abs. 3 BV sowie neue Übergangsbestimmung zu Art. 89 BV; Sammelfrist bis 16.9.2011; .../vi/vis385.html),

- die Volksinitiative „Für ein liberales Rauchergesetz“, welche erreichen will, dass der Eigentümer oder die Eigentümerin eines Lokals wieder selber bestimmen kann, ob geraucht werden darf oder nicht (neuer Art. 118 Abs. 3 und 4 BV; Sammelfrist bis 23.8.2011; .../vi/vis383.html),

- die Volksinitiative „Volkswahl des Bundesrates“, welche die Direktwahl des Bundesrates durch das Volk einführen will (geänderte Art. 136 Abs. 2, Art. 168 Abs. 1, Art. 175 Abs. 2-7 und Art. 176 Abs. 2 BV; Sammelfrist bis 26.7.2011; .../vi/vis380.html),

- die Volksinitiative „Abtreibungsfinanzierung ist Privatsache - Entlastung der Krankenversicherung durch Streichung der Kosten des Schwangerschaftsabbruchs aus der obligatorischen Grundversicherung", die die Finanzierung von Abtreibungen aus dem Leistungskatalog der obligatorischen Krankenversicherung streichen will (neuer Art. 117 Abs. 3 BV sowie neue Übergangsbestimmung zu Art. 117 Abs. 3 BV; Sammelfrist bis 26.7.2011; .../vi/vis381.html),

- die „Familieninitiative: Steuerabzüge auch für Eltern, die ihre Kinder selber betreuen“, welche für solche Eltern einen mindestens gleich hohen Steuerabzug für die Kinderbetreuung fordert wie für Eltern, die ihre Kinder fremd betreuen lassen (neuer Art. 129 Abs. 4 BV; Sammelfrist bis 26.7.2011; .../vi/vis378.html),

- die Volksinitiative „Pädophile sollen nicht mehr mit Kindern arbeiten dürfen“, welche bewirken will, dass wegen Verletzung der Unversehrtheit eines Kindes oder einer abhängigen Person verurteilte Personen keine Tätigkeit mit Minderjährigen oder Abhängigen mehr ausüben dürfen (neuer Art. 123c BV; Sammelfrist bis 20.4.2011; .../vi/vis376.html), sowie

- die Volksinitiative „1:12 - Für gerechte Löhne“, die eine maximale Lohnspannbreite zwischen dem tiefsten und höchsten Lohn in einem Unternehmen von 1:12 in der Verfassung verankern will (neuer Art. 110a BV sowie neue Übergangsbestimmung zu Art. 110a BV; Sammelfrist bis 6.4.2011; .../vi/vis375.html). 
Die Gesetzesrevisionen und referendumspflichtigen Bundesbeschlüsse, die gerade veröffentlicht wurden und 100 Tage lang der Unterschriftensammlung für ein allfälliges fakultatives Referendum unterliegen (Art. 141 Abs. $1 \mathrm{BV}$ ), lassen sich auf der Homepage der Bundeskanzlei abrufen (http://www.ad-min. ch/ch/d/pore $/ \mathrm{rf} / \mathrm{cr} /)^{12}$ In diesem Stadium befinden sich derzeit insgesamt 15 Erlasse. Sie haben überwiegend technischen, nicht politisch-kontroversen Charakter, so dass wahrscheinlich keine weiteren Referenden aus diesem Kreis zustande kommen werden. Da eine Anmeldung zur Unterschriftensammlung nicht erforderlich ist, gibt es allerdings darüber keine offizielle Übersicht.

Im Sammelstadium gescheitert sind

- die Volksinitiative „Verteidigen wir die Schweiz! Das Bankgeheimnis muss in die Bundesverfassung“, mit der die Lockerung des Bankgeheimnisses durch den Bundesrat (März 2009) zurückgenommen werden sollte (neuer Art. 13 Abs. 3-5 BV; Sammelfrist bis 1.10.2010; .../vi/vis369.html),

- das Referendum gegen die Revision des Bundesgesetzes über die technischen Handelshemmnisse (THG), welches sich gegen die einseitige Einführung des „Cassis-de-Dijon-Prinzips“ durch die Schweiz richtete (Änderung zahlreicher Bestimmungen des THG; Ablauf der Referendumsfrist am $1.10 .2009 ; . . . / \mathrm{cr} / 2008 / 20081196 . \mathrm{html})$ und

- die Volksinitiative „68 Milliarden für die soziale Sicherheit“, welche als Reaktion auf die finanziellen Beiträge an die UBS zusätzliche 68 Milliarden für die soziale Sicherheit (AHV, IV, Erwerbsersatzordnung und Arbeitslosenversicherung) fordert (neuer Art. 114a BV sowie neue Übergangsbestimmung zu Art. 114a BV; Sammelfrist bis 26.11.2010; .../vi/vis373.html).

b) Botschaftsphase

Bereits eingereicht, aber mangels Botschaft noch beim Bundesrat hängig (.../vi/vis_1_3_1_2.html), sind derzeit

- die Volksinitiative „Für den öffentlichen Verkehr“, welche die Förderung des öffentlichen Verkehrs erstmals in der Bundesverfassung verankert und die Neuverteilung von Mitteln vorschlägt (neue Art. 81a, 86 Abs. $3^{\text {ter }}$ und 5 BV, geänderte Art. 86 Abs. 3 und 4 BV sowie geänderte Übergangsbestimmung zu Art. $87 \mathrm{BV}$ und neue Übergangsbestimmung zu Art. $86 \mathrm{BV}$; zustandegekommen am 1.10.2010; .../vi/vis366.html),

12 Die dort als Revision erwähnten Gesetze sind in vollständiger Form über ihre Abkürzung in der Systematischen Sammlung zu finden: http://www.admin.ch/ch/d/sr/sr.html. 
- die Volksinitiative „Für eine starke Post“", welche die Sicherstellung eines flächendeckenden Poststellennetzes und einer qualitativ guten Grundversorgung zum Ziel hat (neuer Art. 92 Abs. 3-5 BV; zustandegekommen am 21.9.2010; .../vi/vis377.html),

- die Volksinitiative „Schutz vor Passivrauchen“, die ein grundsätzliches Verbot des Rauchens in allen öffentlich zugänglichen Innenräumen einführen will (neuer Art. 118a BV sowie neue Übergangsbestimmung zu Art. 118a BV; zustandegekommen am 8.6.2010; .../vi/vis371.html),

- die Volksinitiative „Ja zur Hausarztmedizin“, die die Sicherung der Hausarztmedizin in der ganzen Schweiz zum Gegenstand hat (neuer Art. 118b BV; zustandegekommen am 27.4.2010; .../vi/vis374.html), sowie

- die Volksinitiative „Für Geldspiele im Dienste des Gemeinwohls“, mit der die Verwendung von Erträgen aus Geldspielen für das Gemeinwohl (Sport, Kultur und Soziales) konkretisiert werden soll (geänderter Art. 106 BV, neue Art. 106a, 106b BV; zustandegekommen am 6.10.2009; .../vi/vis364.html).

c) Beratungsphase

Vom Bundesrat mit einer Botschaft und Ablehnungsempfehlung versehen und jetzt bei der Bundesversammlung hängig (.../vi/vis_1_3_1_3.html) sind derzeit

- die Volksinitiative „Für die Stärkung der Volksrechte in der Außenpolitik (Staatsverträge vors Volk!)“, welche verlangt, dass völkerrechtliche Verträge in wichtigen Bereichen zwingend dem Volk und den Kantonen zur Abstimmung unterbreitet werden und eine doppelte Mehrheit (Volks- und Ständemehr) erreichen müssen (neuer Art. 140 Abs. 1 Bst. d BV; Botschaft vom 1.10.2010 mit direktem Gegenentwurf; .../vi/vis363.html),

- die Volksinitiative „Sicheres Wohnen im Alter“, mit der Menschen im Pensionsalter bei selbstgenutztem Wohneigentum steuerlich entlastet werden sollen (neuer Art. 108b BV sowie neue Übergangsbestimmung zu Art. 108b BV; Botschaft vom 23.6.2010 mit indirektem Gegenvorschlag; .../vi/vis359.html),

- die Volksinitiative „6 Wochen Ferien für alle“, die die Erhöhung des gesetzlichen Mindestferienanspruchs fordert (neuer Art. 110 Abs. 4 BV sowie neue Übergangsbestimmungen zu Art. 110 Abs. 4 BV; Botschaft vom 18.6.2010 ohne Gegenentwurf; .../vi/vis362.html),

- die Volksinitiative „Raum für Mensch und Natur (Landschaftsinitiative)“, die unter anderem die Vergrößerung des Baugebiets während 20 Jahren verbieten will (geänderter Art. $75 \mathrm{BV}$ sowie neue Übergangsbestimmung zu Art. 75 BV; Botschaft vom 20.1.2010 mit indirektem Gegenvorschlag; .../vi/vis-356.html), 
- die Volksinitiative „Für menschenfreundlichere Fahrzeuge (Stopp-Offroader-Initiative)“, welche die Reduktion der Umweltbelastung sowie den besseren Schutz der Verkehrsteilnehmer durch einen Verkaufsstopp bestimmter Neuwagen und eine Geschwindigkeitsbeschränkung für Altfahrzeuge erreichen will (neuer Art. 82a BV sowie neue Übergangsbestimmung zu Art. 82a BV; Botschaft vom 20.1.2010 mit indirektem Gegenvorschlag; .../vi/vis351.html),

- die Volksinitiative ,jugend + musik“, welche eine Verbesserung der musikalischen Bildung von Kindern und Jugendlichen im obligatorischen Schulunterricht, die Unterstützung von Ausbildungen an Musikschulen sowie die Förderung von musikalisch Begabten anstrebt (neuer Art. 67a BV; Botschaft vom 4.12.2009 ohne Gegenvorschlag; .../vi/vis355.html),

- die Volksinitiative „Eigene vier Wände dank Bausparen“, welche die Förderung des selbstgenutzten Wohneigentums anstrebt (neuer Art. 108a BV sowie neue Übergangsbestimmung zu Art. 108a BV; Botschaft vom 18.9.2009 ohne Gegenvorschlag; .../vi/vis358.html),

- die Volksinitiative „Für ein steuerlich begünstigtes Bausparen zum Erwerb von selbst genutztem Wohneigentum und zur Finanzierung von baulichen Energiespar- und Umweltschutzmaßnahmen (Bauspar-Initiative)“, die das im Kanton Basel-Landschaft praktizierte (und mittlerweile vom Steuerharmonisierungsgesetz nicht mehr zugelassene) Bausparmodell auf nationaler Ebene einführen sowie Hauseigentümer bei energiesparenden Sanierungsmaßnahmen fördern will (neue Art. 129a, 129b BV sowie neue Übergangsbestimmung zu den Artikeln 129a, 129b BV; Botschaft vom 18.9.2009 ohne Gegenvorschlag; .../vi/vis352.html),

- die Volksinitiative „Für ein gesundes Klima“, welche bis im Jahr 2020 eine mindestens 30-prozentige Reduktion der Treibhausgasemissionen durch effizientere Nutzung der Energie sowie durch Förderung von erneuerbaren Energien vorsieht (neuer Art. 89a BV; Botschaft vom 26.8.2009 mit indirektem Gegenvorschlag; .../vi/vis354.html),

- die Volksinitiative „Gegen die Abzockerei“, nach der die Grundsätze für die Lohnpolitik in einer Aktiengesellschaft zukünftig durch die Generalversammlung festgelegt werden sollen (neuer Art. 95 Abs. 3 BV sowie neue Übergangsbestimmung zu Art. 95 Abs. 3 BV; Botschaft vom 5.12.2008 mit indirektem Gegenvorschlag; .../vi/vis348.html), sowie

- die Volksinitiative „Schluss mit uferlosem Bau von Zweitwohnungen!“, welche die Beschränkung des Zweitwohnungsanteils pro Gemeinde auf höchstens 20\% der Bruttogeschossfläche fordert (neuer Art. 75a BV sowie neue Übergangsbestimmungen zu Art. 75a BV; Botschaft vom 29.10.2008 ohne Gegenvorschlag; .../vi/vis345.html).

Offiziell zurückgezogen wurden 
- die Volksinitiative „Gegen maßlosen Bau umwelt- und landschaftsbelastender Anlagen“, die belastende bauliche Anlagen nur noch zulassen wollte, wenn gesamtschweizerisch ein dringendes Bedürfnis dafür besteht (neuer Art. 75 Abs. 4 BV sowie neue Übergangsbestimmungen zu Art. 75 Abs. 4 BV; zurückgezogen am 6.10.2009 zugunsten der inhaltlich ähnlichen Landschaftsinitiative; .../vi/vis344.html),

- die Volksinitiative „Lebendiges Wasser (Renaturierungs-Initiative)“, welche die Wiederherstellung naturnaher Verhältnisse von Fließgewässern forderte (neuer Art. 76a BV sowie neue Übergangsbestimmung zu Art. 76a BV; aufgrund indirekten Gegenvorschlags zurückgezogen am 22.6.2010; .../vi/vis335.html), sowie

- die Volksinitiative „Gegen neue Kampfflugzeuge“, welche ein zehnjähriges Beschaffungsmoratorium forderte (neue Übergangsbestimmung zu Art. 60 BV; zurückgezogen am 13.11.2010; .../vi/vis365.html). Die Initiative wurde zurückgezogen, nachdem der Bundesrat bekannt gegeben hatte, vor 2015 keine Entscheidung über die Beschaffung treffen zu wollen. Eine tatsächliche Durchführung der Beschaffung neuer Kampfflugzeuge ist darum vor Ablauf des geforderten Moratoriums ohnehin äußerst unwahrscheinlich geworden. Auf der offiziellen Seite der Bundeskanzlei (.../vi/vis_2_2_5 4.html) ist der Rückzug bisher noch nicht vermerkt, obgleich er nach übereinstimmenden Äußerungen aller Beteiligter erfolgt sein soll.

d) Abstimmungsphase

Von der Bundesversammlung in ihrer Schlussabstimmung am 1.10.2010 zur Ablehnung empfohlen und damit abstimmungsreif ist

- die Volksinitiative „Für den Schutz vor Waffengewalt“, die Armeewaffen nur noch in gesicherten Räumen aufbewahrt wissen will (geänderter Art. 107 Abs. 1 BV, neuer Art. 118a BV; Botschaft vom 16.12.2009 ohne Gegenvorschlag; .../vi/vis361.html).

\section{Direkte Demokratie in den Kantonen}

1. Grundsätzliche Fragen zur Gültigerklärung kantonaler Volksinitiativen

a) Die Sterbetourismus-Initiative (Zürich)

Im kontrovers diskutierten Bereich der Sterbehilfe, die in der Schweiz grundsätzlich nicht strafbar ist und von den Vereinigungen Dignitas und EXIT in privat 
organisierter Form angeboten wird, stößt vor allem der „Sterbetourismus“ auf breite Ablehnung in der Öffentlichkeit. ${ }^{13}$ Im Kanton Zürich wurde im Berichtszeitraum die Volksinitiative „Nein zum Sterbetourismus im Kanton Zürich!“ lanciert. Sie verlangte in Form einer allgemeinen Anregung, dass der Kanton Rechtsvorschriften erlassen solle, ,welche jegliche Beihilfe zum Selbstmord an Personen ohne mindestens einjährigen Wohnsitz im Kanton Zürich (Sterbetourismus) nicht gestatten und unter Strafe stellen.“ An dieser Volksinitiative lässt sich exemplarisch aufzeigen, wie facettenreich die direkte Demokratie auf kantonaler Ebene gestaltet ist.

\section{b) Ungültigerklärung nur bei offensichtlicher Bundesrechtswidrigkeit}

Die Gültigerklärung auf kantonaler Ebene folgt zwangsläufig anderen Regeln als diejenige bei Initiativen auf Revision der Bundesverfassung. Während eidgenössische Volksinitiativen inhaltlich nur am Maßstab des zwingenden Völkerrechts gemessen werden (Art. 194 Abs. 2 BV), ${ }^{14}$ stehen kantonale Initiativen, selbst wenn sie auf eine Änderung der Kantonsverfassung gerichtet sind, normenhierarchisch unter dem gesamten Bundesrecht. Sie können und müssen für ungültig erklärt werden, wenn sie gegen eine Bestimmung dieses Rechts verstoßen. Abgesehen davon, dass ein Verbot des Sterbetourismus mittelbar das in der Schweiz anerkannte Grundrecht auf Sterben betrifft (,Recht auf den eigenen Tod"), ${ }^{15}$ liegt im Fall der Züricher Sterbetourismus-Initiative möglicherweise ein Verstoß gegen die Kompetenzordnung vor, weil das Strafrecht grundsätzlich zu den Bundeskompetenzen zählt (dazu sogleich).

Als politischer Akt des Parlaments bietet die Gültig- oder Ungültigerklärung allerdings keine Gewähr dafür, dass eine Initiative tatsächlich mit dem Bundesrecht vereinbar ist (oder nicht). Es handelt sich um eine Entscheidung, die gegenüber der endgültigen gerichtlichen Kontrolle nur vorläufig und mit Unsicherheiten belastet ist. In Zürich genügt darum für die Ungültigerklärung einer Volksinitiative nicht bereits die einfache Mehrheit. Vielmehr muss sich eine

13 C. Schwarzenegger u.a., Was die Schweizer Bevölkerung von Sterbehilfe und Suizidbeihilfe hält. Dokumentation zur Medienkonferenz vom 2. September 2010, S. 18 ff. (abrufbar über die Internetseiten der Universität Zürich: http://www.mediadesk.uzh.ch/articles/2010/selbst-bestimmt-ueber-das-lebensende-entscheiden/Bericht_Sterbehilfe.pdf).

14 Zu den Details Tschentscher, Länderbericht 2008/2009 (Fn. 4), S. 214 ff., 216.

15 Dazu A. Tschentscher, Persönliche Freiheit, in: W. Kälin u.a., Die staatsrechtliche Rechtsprechung des Bundesgerichts in den Jahren 2009 und 2010, in: ZBJV 146 (2010), 937 (965 ff., 967 ff.). 
Zweidrittelmehrheit der anwesenden Kantonsratsmitglieder für diese Erklärung aussprechen, sonst ist die Volksinitiative gültig (Art. 28 Abs. 3 KV-ZH). Sinn dieser Regelung ist es, nur die offensichtlich bundesrechtswidrigen Initiativen bereits vor der Abstimmung zu kassieren. Im Übrigen sollen Initiativen, selbst wenn sie im Ergebnis inhaltlich anfechtbar sein sollten, zunächst dem Volk zur Abstimmung unterbreitet werden.

Im konkreten Fall der Sterbetourismus-Initiative hatte sich der Zürcher Kantonsrat (Parlament) nach ausführlicher Diskussion mehrheitlich (98 zu 69 bei drei Enthaltungen) für die Ungültigerklärung ausgesprochen. Die Zweidrittelmehrheit der Anwesenden (141) wurde damit nicht erreicht. Obgleich das Votum relativ deutlich die Skepsis der Parlamentarier hinsichtlich der Bundesrechtskonformität ausdrückt, ist die Initiative damit gültig und muss dem Volk zur Abstimmung vorgelegt werden.

c) Mögliche konkrete Bundesrechtswidrigkeit der Initiative

Ob die Initiative tatsächlich gegen Bundesrecht verstößt, wird man rechtlich bisher als offen ansehen müssen. Immerhin betrifft sie lediglich den Sterbetourismus als Sonderform der Sterbehilfe und dürfte insoweit keine unverhältnismäßige Einschränkung des anerkannten Grundrechts auf Sterben darstellen. Sie könnte allerdings kompetenzwidrig sein. Dem Bund steht die uneingeschränkte Kompetenz für das Strafrecht zu (Art. 123 Abs. 1 BV). Nach der schweizerischen Kompetenzordnung folgt aber aus einer dem Umfang nach unbegrenzten Bundeskompetenz nicht automatisch der vollständige Ausschluss der Kantone von der Gesetzgebung. In der Regel sind Bundeskompetenzen als konkurrierende Kompetenz zu verstehen. Sie verdrängen die kantonale Legislativtätigkeit nur insoweit, als der Bund von der Kompetenz in abschließender Weise Gebrauch macht. Das Strafgesetzbuch belässt den Kantonen ausdrücklich einen Spielraum für die Formulierung von Vergehen (in der Schweiz: „Übertretungen“), soweit das Delikt „nicht Gegenstand der Bundesgesetzgebung ist“ (Art. 335 Abs. 1 StGB). Die Sterbehilfe wird im Strafrecht des Bundes indirekt straffrei gestellt, indem die Hilfe beim Selbstmord (Art. 115 StGB) nur bei ,selbstsüchtigen Beweggründen“ für strafbar erklärt ist. Diese Regelung wird im Verhältnis zu den übrigen Strafandrohungen des Strafgesetzbuches als abschließend angesehen. Damit ist allerdings noch offen, ob die auf individuelle Verantwortlichkeit zielende Straffreiheit auch den Bereich der organisierten Sterbehilfe abschließend erfassen soll. Wenn man in Rechnung stellt, dass bei einem strafbewehrten Verbot des Sterbetourismus, also bloß eines Teils der organisierten Sterbehilfe, die 
individuellen Sterbehilfehandlungen in der Schweiz nach wie vor straffrei blieben, spricht dies für die Zulässigkeit ergänzenden kantonalen Strafrechts in diesem Bereich.

\section{d) Gerichtliche Überprüfung der Gültigerklärung}

Wenn eine Volksinitiative zu Unrecht für gültig erklärt wurde, steht allen Stimmberechtigten des Gemeinwesens dagegen grundsätzlich der Rechtsweg offen. Sie können als Ausdruck ihres eigenen politischen Mitbestimmungsrechts verlangen, dass eine solche Initiative dem Volk überhaupt nicht zur Abstimmung vorgelegt wird. Die Rechtsprechung des Bundesgerichts kennt insoweit allerdings eine bedeutende Einschränkung. Von den Kantonen wird nämlich nicht zwingend gefordert, dass sie Initiativen überhaupt vorgängig auf ihre Vereinbarkeit mit übergeordnetem Recht prüfen. ${ }^{16}$ Es soll zulässig sein, alle Initiativen zunächst zur Abstimmung zu stellen. Eine inhaltliche Rechtswidrigkeit des Begehrens könnte in Kantonen, die eine vorgängige Prüfung nicht vorsehen, dann erst nach der Annahme der Initiative gerichtlich geltend gemacht werden. Die Frage der Gültigerklärung wird also in solchen Fällen von der Frage der Bundesrechtskonformität zeitlich getrennt. Für die Stimmberechtigten stellt dann die Vorlage noch keine Verletzung ihrer Abstimmungsfreiheit dar. Sie müssen vielmehr nach Annahme einer bundesrechtswidrigen Initiative ihr verfassungsmäßiges Recht aus dem Vorrang des Bundesrechts (Art. 49 Abs. 1 BV) gegen das Ergebnis geltend machen.

Diese Rechtsprechung hat das Bundesgericht im Fall der SterbetourismusInitiative auch auf die Gültigerklärung mit qualifizierter Mehrheit übertragen. ${ }^{17}$ Auch bei einem qualifizierten Mehrheitserfordernis wird nach Auffassung des Gerichts hinreichend deutlich, dass der Kanton nicht in jedem Fall eine rechtswidrige Initiative von der Abstimmung ausschließen wolle. Selbst wenn über die Gültigkeit formal befunden wird, ist dieser Entscheid als solcher darum noch nicht anfechtbar. Die Stimmberechtigten in Zürich haben im Ergebnis keinen Anspruch darauf, vor der Abstimmung über rechtswidrige Initiativen verschont zu bleiben. Dasselbe gilt für diejenigen Kantone, die eine Ungültigerklärung nur

16 Ausführlich zur Genese dieser Rechtsprechung seit den 1970er Jahren P. Tschannen, Stimmrecht und politische Verständigung. Beiträge zu einem erneuerten Verständnis von direkter Demokratie, 1995, Rn. 137.

17 BGer-E 1C_92/2010 vom 6. Juli 2010 E. 2.2 - Volksinitiative Sterbetourismus. 
bei „offensichtlicher“ oder ,augenscheinlicher“ Rechtswidrigkeit vorsehen (Basel-Landschaft, Genf). ${ }^{18}$

Diese Rechtsprechung ist in der Literatur nicht ohne Kritik geblieben. Immerhin seien in rechtsstaatlichen Demokratien auch die Volksrechte im Rahmen der Rechtsordnung zu interpretieren; es gelte der Grundsatz: „Keine Behörde sollte deshalb zu Rechtswidrigkeiten Hand bieten.“19 Als Konsequenz dürften dann rechtswidrige Volksabstimmung durch das Parlament überhaupt nicht zur Abstimmung gestellt werden.

Diese Forderung führt - im Ergebnis begrüßenswert - dazu, dass das kantonale Stimmvolk nicht zunächst eine Initiative mehrheitlich annimmt, um dann von den Gerichten wegen Rechtswidrigkeit mit deren Undurchsetzbarkeit konfrontiert zu werden. Gerade bei den völkerrechtswidrigen eidgenössischen Initiativen wird dieses Dilemma der politischen Rechte intensiv diskutiert. Auf kantonaler Ebene stellt sich die Ausgangslage allerdings anders dar. Hier gibt es wegen der komplizierten kompetenziellen Fragen, die sich bei fast jeder Initiative stellen können, die Gefahr einer politisch missbräuchlichen Verwendung der juristischen Einwände. Ein Parlament könnte geneigt sein, rechtliche Gründe vorzuschieben, um politisch missbilligte Vorstöße des Volkes zu umgehen. So gesehen ist es ein legitimes Anliegen, wenn einzelne Kantone ihren politischen Organen nicht die vorgängige Prüfung im Rahmen einer Gültigerklärung zubilligen oder jedenfalls (wie in Zürich, Basel-Landschaft und Genf) das heikle Instrument der Ungültigerklärung auf besonders eindeutige Rechtsverstöße beschränken. Würde man auf die Ausnahmen des Bundesgerichts verzichten wollen, könnte im Ergebnis jede Abstimmung durch jeden Stimmberechtigten vorgängig einer gerichtlichen Kontrolle unterworfen und dadurch zumindest verzögert werden. Dem Kanton wäre ein Gestaltungsrecht genommen und die Entscheidung über die Vorlage zur Abstimmung wäre von politischen Organen auf Gerichtsorgane (Bundesgericht bzw. Verwaltungsgericht ${ }^{20}$ ) übertragen. All dies spricht dafür, dass die Rechtsprechung des Bundesgerichts in diesem Punkt Zustimmung verdient.

18 Dazu Tschannen, Stimmrecht (Fn. 16), Rn. 137 m. Fn. 124 u.w.N.; ders., Staatsrecht der Schweizerischen Eidgenossenschaft, 2. Aufl. 2007, S. 649.

19 Y. Hangartner/A. Kley, Die demokratischen Rechte in Bund und Kantonen der Schweizerischen Eidgenossenschaft, 2000, Rn. 2135 (S. 844 f.).

20 In Bern entscheidet beispielsweise nach dem Gesetz über die politischen Rechte das kantonale Verwaltungsgericht über alle Stimmrechtsbeschwerden in kantonalen Angelegenheiten: Art. 92 Abs. 1 Bst. a GPR-BE. 
2. Grundsätzliche Fragen zur Entwicklung der politischen Mitbestimmung

a) Zukunft der Landsgemeinde

Die Landsgemeinde ist eine der ältesten Formen der direkten Demokratie. Dabei versammeln sich die stimmberechtigten Bürgerinnen und Bürger des Kantons unter freiem Himmel, um bei der Erledigung der legislativen Arbeiten ihrer Stimme per Handaufhalten Ausdruck zu verleihen. Die Stimmenmehrheit wird durch Abschätzen ermittelt. In der Schweiz besteht heute noch in zwei Kantonen (Appenzell Innerrhoden und Glarus) die Landsgemeinde auf kantonaler Ebene. Aufgrund einer Volksinitiative im Kanton Appenzell Außerrhoden wurde im Juni 2010 über die Wiedereinführung der Landsgemeinde abgestimmt, nachdem diese in diesem Kanton im Jahre 1997 (kurz nach Einführung des Frauenstimmrechts) abgeschafft worden war. Die Stimmenden haben mit einer deutlichen Mehrheit die Wiedereinführung der Landsgemeinde abgelehnt.

b) Einführung des Jugendlichenstimmrechts

Das Stimmrechtsalter war neben der Frage des Ausländerstimmrechts erneut in mehreren Kantonen ein Thema. Regelmäßig wurde die Herabsetzung auf 16 Jahre diskutiert. Im Kanton Bern war das Vorhaben ebenso chancenlos wie im Kanton Graubünden. Im Kanton Bern hat das Stimmvolk die Änderung der Kantonsverfassung, welche die Einführung des Stimmrechts sowie des aktiven Wahlrechts ab dem 16. Lebensjahr auf Kantons- und Gemeindeebene vorsah, trotz Empfehlung von Seiten des Großen Rates mit deutlichen 75,4\% Nein-Stimmen abgelehnt. Im Kanton Graubünden wurde nach einem äußerst knappen Entscheid des Großen Rates gegen eine Motion eine Volksinitiative für das aktive Stimmrechtsalter lanciert. Ein Jahr später wurde die Initiative mangels Unterschriften zurückgezogen.

c) Einführung des Ausländerstimmrechts

Zum Ausländerstimmrecht wurden im Berichtszeitraum ebenfalls mehrere Abstimmungen durchgeführt. Die Kantone und Gemeinden sehen bisher verschiedene Varianten des Ausländerstimmrechts vor. Ein Stimm- und Wahlrecht sowohl auf kantonaler als auch kommunaler Ebene gibt es bisher in den Kantonen Neuenburg und Jura. Ein Ausländerstimmrecht nur auf kommunaler Ebene be- 
steht in den Kantonen Waadt, Freiburg und Genf. In den Kantonen Appenzell Außerrhoden, Graubünden und Basel-Stadt können die Gemeinden selber entscheiden, ob sie das Ausländerstimmrecht einführen wollen oder nicht. In diesen Kantonen wurde in einigen Gemeinden das Ausländerstimmrecht verwirklicht.

Ende September 2010 wurde in den Kantonen Bern und Basel-Stadt über die Einführung des Ausländerstimmrechts entschieden. In Basel wurde sowohl über die Volksinitiative „Stimm- und Wahlrecht für Migrantinnen und Migranten“ als auch über einen restriktiveren Gegenvorschlag abgestimmt. Beide wollten das Ausländerstimmrecht auf kantonaler Ebene einführen. Der Gegenvorschlag sah als hauptsächlichen Unterschied zur Initiative keine Wählbarkeit (passives Wahlrecht) von Ausländerinnen und Ausländern vor. Die Volksinitiative wurde mit eindeutigen $80,9 \%$ Nein-Stimmen abgelehnt, beim Gegenvorschlag lag der NeinStimmenanteil bei $61 \%$. Im Ergebnis wurde dadurch das kantonale Ausländerstimmrecht insgesamt abgelehnt.

Im Kanton Bern kam die Volksinitiative ,zäme läbe - zäme schtimme“ (zusammen leben - zusammen stimmen) zur Abstimmung, welche den Gemeinden des Kantons die Möglichkeit geben wollte, auf freiwilliger Basis das kommunale Stimm- und Wahlrecht für Ausländerinnen und Ausländer einzuführen. Das Vorhaben wurde mit 72,3\% Nein-Stimmenanteil ebenfalls deutlich verworfen.

Im letzten Frühling wurde außerdem im Kanton Glarus über die Einführung des Stimm- und Wahlrechts für Ausländerinnen und Ausländer auf kantonaler und kommunaler Ebene abgestimmt. In der Landsgemeinde wurde das Ansinnen auch abgelehnt. Wegen der Stimmenschätzung bei der Landsgemeinde liegen genaue Zahlen hierzu nicht vor.

Im Kanton Zürich haben die Angehörigen der reformierten und römischkatholischen Kirchen im September 2009 über eine Reform ihrer jeweiligen Kirchenordnung abgestimmt, welche als Neuerung das Wahlrecht für Ausländerinnen und Ausländer einführen wollte. Die Vorlage wurde deutlich angenommen (Reformierte Kirche: 75,9\% Ja-Stimmen; Römisch-Katholische Kirche: 78,2\% Ja-Stimmen). Damit können nun in der ganzen Schweiz - Zürich war der letzte Kanton, der entsprechendes Recht eingeführt hat - auch die ausländischen Mitglieder der kirchlichen Gemeinschaft in kirchlichen Angelegenheiten abstimmen und wählen. In der reformierten Kirche haben die Mitglieder außerdem bereits ab dem 16. Lebensjahr das Stimmrecht und das aktive Wahlrecht. 
3. Themen mit mehreren Abstimmungen

Im Übrigen lassen sich für die Kantone unter den zahlreichen Einzelabstimmungen verschiedene Themen identifizieren, zu denen häufig Volksentscheide herbeigeführt wurden.

\section{a) Harmonisierung des Schulwesens}

Die Harmonisierung der obligatorischen Schule Schweiz (HarmoS) auf kantonaler Ebene ist im Berichtszeitraum erneut vorangeschritten. Ein Konkordat ${ }^{21}$ soll die im neuen Bildungsartikel der Bundesverfassung (Art. 62 Abs. 4 BV) verankerte Verpflichtung der Kantone umsetzen. Ziel ist die Vereinheitlichung der wichtigen Eckwerte der obligatorischen Schule für das ganze Land (Verlängerung der Schulpflicht auf elf Jahre, Vorverlegung dieser Pflicht auf das vierte Altersjahr).

Seit dem Zeitpunkt der Vorlage des Konkordats an die Kantone (14.6.2007) wurde über den Beitritt insgesamt in 13 Kantonen durch das Volk abgestimmt. Bei diesen Abstimmungen wurde der Beitritt insgesamt in sieben Kantonen abgelehnt (Luzern, Graubünden, Thurgau, Nidwalden, Uri, Zug, Appenzell Außerrhoden). In den übrigen sechs Kantonen, in denen eine Abstimmung stattfand, wurde das Konkordat angenommen (St. Gallen, Zürich, Bern, Fribourg, Solothurn und Basel-Landschaft). In vier Kantonen steht die Abstimmung noch aus (Aargau, Obwalden, Schwyz, Appenzell Innerrhoden). In den verbleibenden neun Kantonen hat allein das Parlament über den Beitritt entschieden, ohne dass das Referendum ergriffen wurde. Im Ergebnis ist das Konkordat heute in 15 von 26 Kantonen anwendbar. Selbst wenn die vier Kantone, in denen die Abstimmung noch aussteht, zustimmen sollten, nehmen damit von 26 Kantonen sieben definitiv nicht an der Vereinheitlichung teil (27\%).

Eine interessante Entwicklung ergab sich im Kanton Schaffhausen. Nachdem der Kantonsrat als erster den Beitritt zum Konkordat beschlossen hatte und die Referendumsfrist unbenutzt abgelaufen war, traten der kantonsrätliche Beschluss und schließlich das Konkordat in Kraft. Im Dezember 2009 wurde jedoch eine Initiative mit dem Titel „Schaffhausen ohne HarmoS!“ eingereicht, die als Ziel den Austritt des Kantons aus dem Konkordat verfolgte. Bei der Volksabstim-

21 Interkantonale Vereinbarung über die Harmonisierung der obligatorischen Schule (HarmoS-Konkordat) vom 14. Juni 2007. 
mung im November 2010 hat sich das Volk mit knappen 51,7\% gegen die Initiative ausgesprochen. Somit bleibt das Konkordat im Kanton Schaffhausen anwendbar.

b) Nichtraucherschutz

Am 1.5.2010 ist das eidgenössische Bundesgesetz zum Schutz vor Passivrauchen $^{22}$ in Kraft getreten, welches die Grundzüge bezüglich des Nichtraucherschutzes festhält, den Kantonen aber großen Spielraum für weitergehende Beschränkungen lässt, insbesondere das ausnahmslose Rauchverbot in Restaurants. Neben diesem Gesetz wurde ebenso im Mai 2010 die eidgenössische Volksinitiative „Schutz vor Passivrauchen“ eingereicht, welche die Ausnahmen vom Rauchverbot für die ganze Schweiz weiter einschränken möchte.

Auf der Basis der jetzigen Rechtslage wurden im Berichtszeitraum in den Kantonen wiederum mehrere Volksinitiativen und Referenden für oder gegen den Schutz vor Passivrauchen zur Abstimmung gebracht. Sie gingen alle zu Gunsten des Schutzes vor Passivrauchen aus. Im Kanton St. Gallen wurde die Initiative der Lungenliga mit einem Ja-Stimmenanteil von 59\% gutgeheißen. Gegen das neue Gesetz im Kanton Genf ist ein Referendum zustande gekommen, das dann aber in der Volksabstimmung mit nur 18,3\% gegenüber dem Nichtraucherschutzgesetz klar unterlegen ist. In Solothurn hat sich das Stimmvolk mit einem Nein-Stimmenanteil von $66,2 \%$ gegen eine Volksinitiative ausgesprochen, die eine mildere Regelung vorsehen wollte.

Außerdem wurden im Berichtszeitraum mehrere Urteile vom Bundesgericht gefällt. Streitpunkt war jeweils die Zulässigkeit von speziellen Raucherräumen (Fumoirs) in Gastronomiebetrieben. ${ }^{23}$ Das Bundesgericht hat in jedem der Fälle das Rauchverbot geschützt und keine Verletzung von Grundrechten festgestellt. Insbesondere wurde durch die kantonalen Regelungen die Wirtschaftsfreiheit nicht verletzt.

22 Bundesgesetz zum Schutz vor Passivrauchen vom 3.10.2008, SR 818.31.

23 BGE 136 I 29 - Fumoir Bern; BGE 136 I 17 - Wasserpfeifen; BGer-E 2C_117/2010 vom 17. August 2010 - Fumoir Solothurn I; BGer-E 2C_233/2010 vom 17. August $2010-$ Fumoir Solothurn II. 
c) Ladenöffnungszeiten

Nachdem das Stimmvolk in Bund und Kantonen vereinzelt für eine Lockerung der Ladenöffnungszeiten plädiert hatte - insbesondere anlässlich einer eidgenössischen Abstimmung im Jahr 2005, mit der die Öffnungszeiten für Verkaufsgeschäfte an Flughäfen und Bahnhöfen liberalisiert wurde -, scheiterten im Berichtszeitraum gleich mehrere Abstimmungen auf kantonaler Ebene. Die Bedenken der Liberalisierungsgegner, dass sich die Arbeitsbedingungen für das Verkaufspersonal verschlechtern und das Kleingewerbe leiden würden, wurden offenbar für gewichtiger gehalten als die Vorzüge der längeren Öffnungszeiten für Konsumenten. Im Kanton Fribourg hat sich das Stimmvolk aufgrund eines von den Gewerkschaften gegen die Revision des Handelsgesetzes ergriffenen Referendums mit 57,7\% Nein-Stimmen gegen längere Ladenöffnungszeiten ausgesprochen. Ebenso wurde im Kanton Uri eine Verlängerung der Öffnungszeiten abgelehnt. Das Stimmvolk hat sich mit deutlichen 69,4\% Nein-Stimmen gegen die Revision des Gesetzes über den Ladenschluss und die Sonntagsruhe ausgesprochen. Desgleichen hat das Volk im Kanton Genf die Änderung des kantonalen Gesetzes über die Geschäftsöffnungszeiten mit 56,2\% Nein-Stimmen verhindert. Gegen das Gesetz, welches ebenfalls längere Öffnungszeiten zulassen wollte, war das Referendum ergriffen worden.

\section{d) Energiegesetze}

Über kantonale Energiegesetze wurde im Berichtszeitraum vermehrt abgestimmt. In Schwyz wurde das neue Energiegesetz mit einem Ja-Stimmenanteil von $67,8 \%$ angenommen. Es sieht energiesparende Vorschriften für Neubauten sowie Anreize für die Nutzung von erneuerbaren Energien vor. Gleichzeitig wurde die Volksinitiative „Für eine aktive Schwyzer Energiepolitik - Mehr Energieeffizienz und erneuerbare Energien“, die eine weitergehende Förderung verlangte, von den Stimmenden mit 53\% Nein-Stimmen abgelehnt.

Im Kanton Neuenburg wurde das neue Energiegesetz von den Stimmenden deutlich mit 65\% Nein-Stimmen abgelehnt. Mit dem Gesetz wollte der Kanton ganz auf erneuerbare Energien setzen, Neubauten nur noch mit Solaranlagen und Wärmepumpen ausgerüstet sehen und bestehende Häuser an Wärmenetze angeschlossen haben. 
e) Steuerpakete

Eine Änderung des Gesetzes über die Staats- und Gemeindesteuern im Kanton Thurgau, welche die Einführung eines proportionalen Einheitssteuersatzes von $6 \%$ vorsah, wurde vom Stimmvolk mit einem Nein-Stimmenanteil von 54,7\% verworfen. Damit bleibt es bei zwei Kantonen (Obwalden, Uri) in der Schweiz mit einer Flat-Rate-Tax.

Über Steuerpakete wurde auch in drei weiteren Kantonen abgestimmt. Diese wurden sowohl in Luzern, als auch in Basel-Landschaft und Genf gutgeheißen. In Luzern wurde die Gewinnsteuer gesenkt. Der Kanton Basel-Landschaft senkte die Erbschafts- und Schenkungssteuer und gewährt nun Unternehmen steuerliche Erleichterungen. Bei der Abstimmung im Kanton Genf stand die Besteuerung von verheirateten Paaren im Vordergrund. Diese werden aufgrund des VollSplittings künftig unverheirateten Paaren gleichgestellt. Bisher wirkte sich die Heirat im Vergleich zur nichtehelichen Lebensgemeinschaft steuerlich nachteilig aus.

4. Weitere prominente Einzelthemen

a) Familienergänzende Kinderbetreuung

Auf kantonaler Ebene wurde in Zürich die Volksinitiative „Kinderbetreuung Ja“ eingereicht, welche die familienergänzende Kinderbetreuung als öffentliche Aufgabe gesetzlich verankern wollte. Kanton und Gemeinden sollten gemeinsam für ein nachfragegerechtes Angebot für Kinder im Vorschul- und im Schulbereich sorgen, während die Finanzierung über Elternbeiträge und Beiträge von Kanton und Gemeinden erfolgen sollte. Der Kantonsrat hatte einen Gegenvorschlag ausgearbeitet, der weniger weit ging und an die für den Volksschulbereich bereits bestehenden Regelungen zu den familien- und schulergänzenden Tagesstrukturen anknüpfte. Dabei hätten Gemeinden verpflichtet werden sollen, ein bedarfsgerechtes familienergänzendes Angebot für Kinder im Vorschulalter zu schaffen. Den Gemeinden sollte freigestellt bleiben, welche Ausgestaltung und Finanzierung sie vorsehen wollten. Entsprechend der Empfehlung des Kantonsund Regierungsrats hat das Stimmvolk die Initiative mit 65,5\% Nein-Stimmen verworfen und den Gegenvorschlag mit 62,4\% Ja-Stimmen angenommen. 
b) Fluglärm

Eine erneute Diskussion zur Fluglärmproblematik rund um Zürich entfachte die Volksinitiative „Für eine faire und ausgewogene Verteilung des Fluglärms um den Flughafen Zürich (Verteilungsinitiative)“. Die Initiative strebte eine Verteilung der Flugbewegungen an. Die Abflüge sollten möglichst in die Richtung der Destination erfolgen, während Landungen grundsätzlich von Norden her erfolgen sollten. Das Ansinnen wurde vom Kantons- und Regierungsrat abgelehnt. Entsprechend hat sich das Stimmvolk mit einem Nein-Stimmenanteil von 75,2\% gegen die Vorlage entschieden. Den Hintergrund dazu bildet der Umstand, dass die Initianten vorwiegend aus einer einzelnen Region stammten und ihre Aktivitäten während des Abstimmungskampfes aus finanziellen Gründen weitgehend einschränken mussten.

c) Leistungslohn

Im Kanton Tessin wurde dem Stimmvolk aufgrund eines Referendums die Gesetzesrevision über die Einführung des Leistungslohns für Kantonsangestellte zur Abstimmung vorgelegt. Die Revision sah vor, dass Kantonsangestellte nicht mehr entsprechend ihrem Dienstalter automatisch Lohnerhöhungen erhalten sollten, sondern dass ein bestimmter Prozentsatz des Lohnes als Prämie für gute Leistungen gezahlt würde. Der Entscheid ist mit 54,1\% Nein-Stimmenanteil gegen die Revision ausgefallen.

d) Kampfhundeverbot

Anknüpfend an die im letzten Bericht erwähnten erfolgreichen Volksabstimmungen über Kampfhundeverbote, kam es diesbezüglich zu einem Bundesgerichtsentscheid. Gegen die im Kanton Zürich vom Volk gutgeheißene Regelung wurde von Hundezüchterkreisen Beschwerde beim Bundesgericht eingereicht. Das zürcherische Hundegesetz setzt bei der Hunderasse an. Diesbezüglich erkannte das Gericht keinen Verstoß gegen übergeordnetes Recht. Außerdem hielt das Bundesgericht fest, dass die Würde der Tiere nicht durch die Regelung ver- 
letzt werde und dass ebensowenig eine Verletzung der Wirtschaftsfreiheit vorliege. $^{24}$

\section{Direkte Demokratie in den Gemeinden}

\section{Grundsätzliche Fragen}

Der inhaltliche Umfang der kommunalen Volksabstimmungen variiert kantonal mit dem unterschiedlichen Umfang der gemeindlichen Kompetenzen. Die Anzahl der im Berichtszeitraum erfolgten kommunalen Mitwirkungsakte des Volkes ist derart groß, dass sich dieser Bericht auf einige politisch brisante Schwerpunkte beschränkt. Über diese Schwerpunkte hinaus wurde in den Gemeinden vor allem über baurechtliche, polizeirechtliche und steuerrechtliche Fragen abgestimmt. Zudem fanden nach bejahenden Volksabstimmungen Gemeindefusionen statt.

2. Abstimmungspraxis

a) Reitschule Bern

Die Reitschule Bern ist ein autonomes Kultur- und Begegnungszentrum in der Stadt Bern. Neben dem Betrieb diverser Restaurations- und Barlokale finden Konzerte, Theateraufführungen und Ausstellungen statt. Aufgrund verschiedener Vorkommnisse (insbesondere Gewalttaten) war die Reitschule immer wieder Gegenstand politischer Diskussionen. Seit ihrem 20jährigen Bestehen hat das Berner Stimmvolk bereits fünf Mal über die Zukunft der Reitschule abgestimmt:

$\mathrm{Zu}$ Beginn der Neunziger Jahre wurde eine erste Initiative zur Abstimmung vorgelegt. Diese forderte den Abbruch der Reitschule und die Errichtung eines Sportzentrums an deren Stelle. Das Vorhaben wurde am 2.12.1990 mit einem Nein-Stimmenanteil von $57,6 \%$ abgelehnt.

Ende der Neunziger Jahre kam es zu einer Volksabstimmung über die Finanzierung der Sanierung der Reitschule. Der äußerst knappe Entscheid ging am 13.6.1999 mit 50,1\% Ja-Stimmen zu Gunsten der Sanierungsvorlage aus. 
Die Initiative „Reitschule für alle“ strebte den Betrieb eines Einkaufzentrums mit Laden-, Gewerbe- und Dienstleistungsnutzungen sowie die Errichtung einer Parkieranlage auf dem Areal der Reitschule an. Das Volk hat am 24.9.2000 auch dieses Vorhaben mit 67\% Nein-Stimmen abgelehnt.

Über eine weitere Initiative bezüglich der Reitschule wurde am 27.11.2005 abgestimmt. Die Initiative „Keine Sonderrechte für die Reitschule“ verlangte, dass die Reitschule ortsübliche Mietzinse und Nutzungsgebühren bezahlen sowie alle notwendigen Bewilligungen einholen müsse. Sie wurde mit einem NeinStimmenanteil von 65\% durch das Berner Stimmvolk abgelehnt.

Vor diesem kontroversen Hintergrund wurde am 26.9.2010 zum fünften Mal über einen Vorstoß bezüglich der Reitschule Bern abgestimmt. Die Initiative „Schließung und Verkauf der Reitschule“ forderte den Verkauf an den Meistbietenden sowie die Umnutzung des Areals. Mit bloß 31,6\% Ja-Stimmen wurde auch dieses Ansinnen vom Volk abgelehnt.

\section{b) Atomausstieg}

Nachdem im Jahre 2008 in der Stadt Zürich der schrittweise Atomausstieg beschlossen worden war (76,4\% Ja-Stimmen beim obligatorischen Referendum zur Änderung der Gemeindeverfassung), hat sich im November 2010 das Stimmvolk der Stadt Bern diesbezüglich geäußert. Aufgrund einer Volksinitiative „EnergieWende-Bern“ mit stadträtischem Gegenvorschlag wurde darüber abgestimmt, ab wann die Stromproduktion und der Stromverkauf ausschließlich aus erneuerbaren Energien erfolgen solle. Die Initiative sah eine 20jährige Frist vor, während der Gegenvorschlag zur Kostendämpfung das Ziel erst bis 2039 erreicht sehen wollte. Der Stadtrat hatte die Initiative zur Ablehnung und den Gegenvorschlag zur Annahme empfohlen. Entsprechend hat sich das Stimmvolk geäußert. Der Gegenvorschlag wurde mit 60,6\% Ja-Stimmen angenommen und die Initiative mit 51,2\% Nein-Stimmen abgelehnt.

Gleichzeitig fand auch in der Stadt St. Gallen eine Volksabstimmung über die Volksinitiative „Stadt ohne Atomstrom“ und den Gegenvorschlag des Stadtparlaments zum schrittweisen Ausstieg aus der Atomenergie statt. Initiative und Gegenvorschlag verfolgten das Ziel, die Versorgung der Stadt St. Gallen mit erneuerbaren Energien zu fördern und schrittweise aus der Atomenergie auszusteigen. Mit der Initiative wurde die Einschränkung der Handlungsfreiheit der Stadt bei der Energiebeschaffung angestrebt, indem die Stadt keine AtomstromVerträge mehr verlängern oder neu hätte abschließen dürfen. Der Gegenvorschlag wollte basierend auf dem städtischen Energiekonzept und unter Wahrung 
der Versorgungssicherheit mit einem Energie-Artikel in der Gemeindeordnung den schrittweisen Ausstieg bis 2050 erreichen. Die Initiative wurde mit 58,6\% Nein-Stimmen abgelehnt, der Gegenvorschlag hingegen mit 61,4\% Ja-Stimmen angenommen.

Die Abstimmungen könnten einen Vorgeschmack auf kommende Volksabstimmungen zum Thema Kernkraft geben. Auf kantonaler Ebene wird im Februar 2011 im Kanton Bern über den Ersatz des Kernkraftwerks Mühleberg abgestimmt. In einigen Jahren wird eine nationale Abstimmung zur Atomenergie stattfinden.

\section{c) Gewalt an Sportveranstaltungen}

Anlässlich der Euro 2008 wurde die Hooligan-Datenbank HOOGAN in Betrieb genommen. In dieser Datenbank werden Personen erfasst, die sich bei Sportveranstaltungen im In- und Ausland gewalttätig verhalten haben und gegen die Maßnahmen wie Stadion- und Rayonverbote, Meldeauflagen, Polizeigewahrsam oder Ausreisebeschränkungen verhängt wurden. Diese im Bundesgesetz über Maßnahmen zur Wahrung der inneren Sicherheit befristete Regelung wurde durch ein interkantonales Konkordat auf Initiative der Konferenz der kantonalen Justiz- und Polizeidirektorinnen und -direktoren (KKJPD) abgelöst. Mit dem Hooligan-Konkordat ermächtigen die Kantone im Rahmen ihrer Polizeikompetenz den Bund zur Weiterführung der schon bestehenden Datenbank.

Nachdem der Kanton Zürich dem Konkordat bereits beigetreten ist, beschloss der Gemeinderat der Stadt Zürich (Parlament) zusätzlich eine Verordnung, welche die Inbetriebnahme einer Datenbank GAMMA vorsieht, die in ganz ähnlicher Weise gegen Fangewalt gerichtet ist. In der Datenbank werden solche Personen registriert, welche die Nähe zur Gewalt suchen oder gewaltbereit sind. Folglich findet die Registrierung bereits statt, bevor eine Straftat begangen wurde - dies im Gegensatz zur HOOGAN-Regelung, welche eine Datenspeicherung erst nach gewalttätigem Verhalten vorsieht. Die betroffenen Personen werden über die Registrierung informiert. Die Parlamentsverordnung wurde infolge eines Referendums dem Stimmvolk zur Annahme vorgelegt. Das Stimmvolk hat dem Vorhaben mit einem sehr hohen Ja-Stimmenanteil von 72,6\% zugestimmt. Die Datenbank wurde vorläufig für ein Jahr in Betrieb genommen und wird bloß bei erwiesener Wirksamkeit (Abschreckungswirkung) weitergeführt. 
Abgekürzte Links:

.../as/ = www.admin.ch/ch/d/as/

.../ff/ = www.admin.ch/ch/d/ff/

.../geschaefte.aspx $=$ www.parlament.ch/d/suche/seiten/geschaefte.aspx

.../ref/ = www.bk.admin.ch/aktuell/abstimmung/ref/

.../cr/ = www.admin.ch/ch/d/pore/rf/cr/

.../sr/ = www.admin.ch $/ \mathrm{ch} / \mathrm{d} / \mathrm{sr} /$

.../va/ = www.admin.ch/ch/d/pore/va/

.../vi/ = www.admin.ch/ch/d/pore/vi/ 